\title{
The effect of offspring value on variation of nest defense intensity in gray catbirds (Dumetella carolinensis)
}

\author{
Lucas J. Redmond ${ }^{1}$ (1) $\cdot$ Craig Eroh $^{1} \cdot$ Jordan I. Haq ${ }^{1} \cdot$ Dominique Varra $^{1}$
}

Received: 26 September 2019 / Revised: 29 January 2020 / Accepted: 5 February 2020 / Published online: 20 February 2020

(C) The Author(s) 2020

\begin{abstract}
Nest defense is an adaptive strategy used by adults to increase survival of independent young. However, the risk adults face by defending young from predators represents an important trade-off and parental investment theory predicts adults will modulate nest defense based on the value of offspring. We used a model of a black rat snake, a potential nest predator on our study site, to quantify nest defense in a population of gray catbirds in eastern Pennsylvania during the breeding seasons of 2017 and 2018. Specifically, we evaluated (1) the difference in nest defense between male and female parents, (2) the relationship between nest defense and nest success, and (3) how variation in nest defense was related to factors associated with offspring value. Although male catbirds tend to be larger than females, they did not defend their nests more aggressively than female catbirds. Response to the model predator we used was also not different between individuals whose nests were successful compared with individuals that failed. Although nests that contained more and older young were not defended more aggressively, our analyses did show that adult catbirds defended young that were laid earlier in the season more intensely than those laid later in the breeding season. In birds, earlier clutch initiation is associated with an increased chance of recruitment into the breeding population. Catbirds, therefore, follow predictions of parental investment theory because they defended offspring of higher value more aggressively.
\end{abstract}

\section{Significance statement}

Defense of young from predators is an important component of parental care. Nest defense represents a trade-off because although it should increase reproductive success by increasing offspring survival, it presents a risk to adults, thus lowering their survival. We studied variation in nest defense of gray catbirds in relation to offspring value. Although nest defense was not related to nest success, we did find that adult catbirds defended nests that were produced earlier in the year more aggressively than later nests. In birds, the timing of reproduction is linked to prospects of juvenile survival in such a way that offspring produced early in the breeding season are more likely to survive and recruit into the breeding population. As predicted by parental investment theory, catbirds defended young that represented a higher increase in fitness more aggressively.

Keywords Nest defense $\cdot$ Parental investment $\cdot$ Parental care $\cdot$ Catbird $\cdot$ Nest predator

\section{Introduction}

Many vertebrates, especially birds, mammals, and some fishes, will attempt to defend their young from predators (CluttonBrock 1991). Birds, for example, are known to use a variety of

Communicated by M. Leonard

Lucas J. Redmond

ljr5322@psu.edu

1 Department of Biology, Penn State Schuylkill, 200 University Dr., Schuylkill Haven, PA 17972, USA nest defense mechanisms that range from distraction displays (Brunton 1990) to physical aggression towards potential predators (Davis et al. 2018). Within a species, the intensity with which adults defend their offspring will vary and explanations for this are usually based on parental investment (PI) theory in relation to the perceived value of offspring to parents (Trivers 1972; Montgomerie and Weatherhead 1988). From a fitness perspective, the number of eggs and/or nestlings is perhaps the most straightforward example of how nests could vary in their perceived value to adults and several studies have shown that nest defense intensity is positively related to the number of offspring in nests (Knight and Temple 1986a; Olendorf and Robinson 2000; Jonsson et al. 2002; Svagelj et al. 2012; but 
see Graham and Shutler 2019). Although nest defense may impart a fitness benefit by increasing prospects of offspring survival, it is not without cost because, like other forms of parental care, defending adults could experience reduced survival (Visser and Lessells 2001) or fecundity (Hanssen et al. 2005).

A second factor that is expected to cause variation in perceived value is offspring age because older offspring are more likely to reach independence than younger offspring (Dawkins and Carlisle 1976; Boucher 1977). Therefore, parents are expected to defend older offspring more aggressively (e.g., Andersson et al. 1980; Tryjanowski and Golawski 2004; Redmond et al. 2009; Svagelj et al. 2012). Studies in a number of species have shown that young who fledge earlier in the breeding season exhibit higher rates of juvenile survival (survival until the beginning of the first breeding season) compared with young that fledge from nests later in the breeding season (Drilling and Thompson 1988; Verhulst et al. 1995; Shutler et al. 2006). Because earlier fledging offspring are more likely to survive to reproduce, PI theory also predicts that adults should defend those young more aggressively (Redmond et al. 2009; Boukhriss and Selmi 2010; Morrell et al. 2016). However, other studies have found the opposite and suggest that a decline in renesting potential, the likelihood that an adult will attempt to renest following nest failure, across the breeding season results in an increase in nest defense intensity with date in the breeding season (Montgomerie and Weatherhead 1988; Pavel and Bures 2008). An increase in nest defense intensity throughout the season could also be caused by the same individuals being repeatedly exposed to a model predator across the duration of a study, potentially resulting in positive reinforcement of this behavior, and thus, more aggressive nest defense later in the season (Knight and Temple 1986b).

Variation in nest defense could also be related to traits of the parent that is defending the nest. For example, males of most species studied defend their nests more vigorously than females (Regelmann and Curio 1986; Redondo 1989; Redmond et al. 2009), which could be a result of males being larger than females making them less prone to injury when attacking a predator and allowing them to be more aggressive while defending their nest (Montgomerie and Weatherhead 1988; Kis et al. 2000) or more efficient, because of a larger body size, at defending nests (Wallin 1987).

We studied variation in nest defense behavior in gray catbirds (Dumetella carolinensis, hereafter catbirds). Catbirds are multiple-brooded, socially monogamous, open-cup nesting songbirds that exhibit bi-parental care. Thus, they are wellsuited to study predictions of PI theory, especially as they relate to associations between seasonal timing and perceived value of offspring. We conducted experimental observations of nest defense behavior by catbirds over the course of two breeding seasons using a model of a black rat snake
(Pantherophis obsoletus). This species of snake is a frequently encountered predator that has been shown to prey on both eggs, nestlings (Thompson III et al. 1999), and adult birds (Stickel et al. 1980) and represents a viable risk to adults defending their offspring. Therefore, we hypothesized that variation in catbird nest defense would follow predictions of PI theory and predicted that catbirds would defend nests of higher perceived value more aggressively than nests that were less valuable. We predicted that nest defense intensity would increase with both the number and age of offspring in the nest at the time of observation. Like most species, clutch size in the population we studied was inversely related to clutch initiation date (LJR unpublished data). We also predicted that nest defense intensity would decline throughout the season as catbirds were expected to defend smaller broods as the season progressed. Catbirds in the population we studied will usually attempt to raise at least two broods per year, with peaks in fledgling production occurring in early June and again in late June and early July. Finally, because of the expected decline in offspring recruitment with season, we predicted that catbirds would defend earlier broods more aggressively than later broods.

\section{Methods}

\section{Study site and species}

This study was conducted on and around the Penn State Schuylkill campus located in Schuylkill Haven, Pennsylvania $\left(40.6393^{\circ} \mathrm{N}, 76.1673^{\circ} \mathrm{W}\right)$, during the breeding seasons of 2017 and 2018. Our study site was approximately 45 ha of land that primarily consisted of second growth forest with a shrubby understory of multiflora rose (Rosa multiflora), tatarian honeysuckle (Lonicera tatarica), and privet (Ligustrum sp.) which were the most commonly used shrub species by catbirds for nesting (LJR unpublished data). Beginning in mid-May of each year, we conducted daily nest searches throughout the study site. Nests were located by thoroughly searching suitable habitat and observing parental behaviors. Nest location was recorded on a global positioning system and nests were observed throughout the nest cycle. Nests were checked every third or fourth day until they either failed or when nestlings were 10 days old and banded, at which point we considered nests to be successful. We attempted to capture as many adults as possible using mist nets placed around nests when nestlings were between 5 and 9 days old. Once captured, adults were banded with a unique combination of one aluminum USFWS band and three colored, plastic leg bands and a small $(150-200 \mu \mathrm{L})$ blood sample was taken. We measured the length, width, and depth of the bill and tarsus length with dial calipers; wing chord and tail lengths with a stopped rule; and body mass with a spring scale. 


\section{Behavioral observations}

We simulated predation events on catbird nests with a $1.1-\mathrm{m}$ long replica of a black rat snake, an often-encountered predator known to depredate bird nests. Given that Gottfried (1979) showed that catbirds responded aggressively to predators and not to a control, we assumed that catbirds perceived the snake as an actual predator and did not include a control in this experiment. This assumption was supported by trail camera footage (LJR unpublished data) showing that catbirds responded to real predators in a manner consistent with what we observed in this study. The predator was placed within $1 \mathrm{~m}$ of the focal nest, while two nearby observers (approximately 15 to $25 \mathrm{~m}$ away, depending on understory density and nest visibility) concealed themselves as much as possible. Once positioned, the person that placed the predator moved away from the nest and a 30-min countdown for parental response began. To distinguish between the males and females at the nest, we only conducted observations at nests where at least one of the adults had been previously banded; thus, the identity of at least one adult at each nest was known. Because of this, it was not possible to record data blind because our study involved focal animals in the field. We eliminated scoring bias between the sexes based on observer differences by randomly assigning the male and female at a nest to one of the two observers. If at least one of the focal adults responded in a manner associated with defending the nest against a predator during the 30-min period, we recorded the elapsed time since the placement of the predator (hereafter, response time) and the observer began recording the behaviors exhibited by adults for the next $5 \mathrm{~min}$. At the end of the $5 \mathrm{~min}$, or if adults failed to either find or respond to the predator during the 30min period, the predator was removed. The presence of predators can elicit three different vocalizations in catbirds which are best described as (1) the "mew" call, (2) the "quirt" call, and (3) the ratchet call (Smith et al. 2011), and we recorded the number of times each of these vocalizations were given by adults. Catbirds also approach predators and raise and quiver one or both of their wings. Finally, catbirds will attack predators to drive them away from the nest. We recorded the number of times each of the three vocalizations, wing raises, and attacks were observed during the 5-min recording period following initial discovery and response to the predator by at least one of the adult catbirds.

\section{Statistical analyses}

We quantified catbird response to predators by calculating a single value to represent how aggressively individuals behaved towards the predator during the 5-min scoring period. First, we assumed that vocalizations in response to the predator were a less aggressive form of nest defense than either wing raises or attacking the predator. We also assumed that attacking the predator exhibited more aggression and represented a higher level of risk than a wing-raise in response to the predator. Therefore, similar to previously published studies that used a single value to quantify how aggressively individuals defend their nests from predators (Curio and Onnebrink 1995; Redmond et al. 2009; Krystofkova et al. 2011), we modified the value of these behaviors with the following equation which was used to calculate nest defense intensity (NDI):

$\mathrm{NDI}=(($ Mews + Quirts + Ratchets $) \times 0.5)+($ Wing raises $\times$ 2) $+($ Attacks $\times 3)$

Because of field assistant turnover both within and between years, nest defense observations were made by six different observers. The number of different observers that collected data such as those used in this study could lead to biased results because of differences in recording behaviors. To test for observer bias in scoring, we compared NDI among the six observers with an ANOVA. We further tested for betweenyear biases caused by observer turnover by comparing NDI between years using a $t$ test.

Our analyses to determine important sources of variation in NDI began by testing for differences in NDI and response time between the sexes using $t$ tests. This was done at both a population level and on a pairwise basis. We assumed that nest defense is an adaptive behavior for catbirds and predicted that nests that were either more aggressively or vigilantly defended would be more likely to fledge offspring. We tested these predictions by comparing NDI and response times of nests that were successful to those that failed using $t$ tests.

We used an information theoretic approach to determine the effect of variables associated with offspring value as predicted by PI theory on NDI. These variables included observation date, clutch initiation, day in the nest cycle, brood (first or later), stage of the nest cycle, and nest worth, which was calculated as the number of offspring present in the nest at the time of observation, adjusting for an assumed difference in perceived value between eggs and nestlings by multiplying the number of nestlings by two. In an earlier analysis, we distinguished between second broods (first nest of pair was successful) and replacement nests (first nest of pair failed). However, we found no difference in NDI scores between these nests and to avoid overparameterization in analyses we grouped them together as later broods. We also included sex, the length of the bill and tail, and body condition variable that was derived from residuals of a linear regression of body mass on tarsus length for individuals that were observed. We used general linear models to determine the effect that these variables had on variation in NDI. In these models, brood, stage, and sex were included as fixed factors, while observation date, clutch initiation, day in the nest cycle, and nest worth were included as covariates. Because of sample size concerns, we only considered models with a maximum of four predictor variables. Akaike information criterion corrected for small 
sample size $\left(\mathrm{AIC}_{\mathrm{c}}\right)$ was determined for each candidate model, which we used to calculate model likelihoods and weights (Burnham and Anderson 2002). We considered models that were within $2 \mathrm{AIC}_{\mathrm{c}}$ units of the model with the lowest $\mathrm{AIC}_{\mathrm{c}}$ score as being competitive. Weights of competitive models were used to calculate model-averaged parameter estimates for regression coefficients and their standard errors and 95\% confidence intervals of predictor variables (Burnham and Anderson 2002; Arnold 2010). Variables that did not include zero between the upper and lower confidence intervals of their regression coefficients were deemed important contributors to variation in NDI scores. Some individuals were observed multiple times and to avoid pseudoreplication we randomly selected a single case for those individuals to include in analyses. Values are reported as means $\pm \mathrm{SE}$ and all analyses were conducted in SPSS 25 (SPSS 2017).

\section{Data availability}

The datasets generated and/or analyzed during the current study are available from the corresponding author on reasonable request.

\section{Results}

\section{General patterns}

Although six different observers were involved over the 2 years of this study, mean NDI among these observers did not differ $(F=0.69, P=0.63)$. We conducted 76 trials in 2017 and 36 trials in 2018. There was no difference in mean NDI values between years $(2017,0.68 \pm 0.10 ; 2018,0.72 \pm 0.15$; $t=-0.19, P=0.85$ ), regardless of whether analyses included the sexes together or separately (males: $P=0.44$; females: $P=$ $0.27)$.

Males had longer bills than females (male, 11.87 \pm $0.14 \mathrm{~mm}, n=32$, female, $11.46 \pm 0.12 \mathrm{~mm}, n=35 ; t=$ 2.22, $P=0.03$ ) and tended to have longer tails than females (male, $95.81 \pm 0.87 \mathrm{~mm}, n=32$, female, 93.54 $\pm 0.87, n=35 ; t=1.84, P=0.07)$. At the population level, male and female NDI scores (male, $0.76 \pm 0.16, n=$ 32, female, $0.96 \pm 0.14, n=35 ; t=0.96, P=0.34)$, and response time between males $(13.6 \pm 9.9 \mathrm{~min}, n=17)$ and females $(12.2 \pm 7.9 \mathrm{~min}, n=25 ; t=0.50, P=0.62)$ did not differ. Within pairs, male and female NDI (male, $1.07 \pm 0.16, n=32$, female, $1.34 \pm 0.14, n=32$; $t=-1.62, P=0.12)$ was also not different and neither was the time it took for paired males $(13.5 \pm 2.0 \mathrm{~min}$, $n=24)$ and females $(13.5 \pm 2.0 \mathrm{~min}, n=24 ; t=-0.53$, $P=0.60)$ to respond. Instead, both NDI $(r=0.45, P=$ $0.007)$ and response time $(r=0.99, P<0.001)$ were highly correlated between paired males and females.
In 45 of the 112 observations, adults did not appear at the nest during the entire 30-min observation period. An additional 15 cases resulted in adults at the nest that either (1) did not respond to the predator or (2) failed to find the predator during the 30-min observation period. We classified cases as nonresponses when adults were in close proximity to the predator and appeared to have the predator within their visual field yet did not exhibit behaviors typical of nest defense. Of the 52 cases that elicited a response by an adult, the mean time to find and respond to the predator was $12.0 \pm 1.2 \mathrm{~min}$. Time to respond did not vary with observation date $(F=0.01, P=0.97)$, clutch initiation date $(F=0.01, P=0.97)$, and offspring age $(F=0.30, P=0.59)$ or nest worth $(F=0.57, P=0.45)$. NDI scores between successful $(0.92 \pm 0.17, n=21)$ and failed nests $(0.84 \pm 0.13, n=46 ; t=0.36, P=0.72)$ were not different and adults whose nests were ultimately successful (15.6 \pm $2.1 \mathrm{~min}, n=16$ ) did not respond to the predator faster during trials than those adults whose nests failed $(11.1 \pm 1.7 \mathrm{~min}, n=$ $26 ; t=1.67, P=0.10$ ).

\section{Offspring value and nest defense}

Results of model selection (Table 1) indicated that the model with the lowest $\mathrm{AIC}_{\mathrm{c}}$ value included only clutch initiation as a predictor. Six other models had $\Delta \mathrm{AIC}_{\mathrm{c}}$ values less than 2.0, but they all included clutch initiation in addition to some other variable or combination of variables. Because there were several competing models, we used model-averaging to produce parameter estimates of slope coefficients and their standard errors and $95 \%$ confidence intervals for all predictor variables used in model selection. Based on the calculated confidence intervals (Table 2), clutch initiation was the only predictor that exhibited an important, and negative, effect on NDI (Fig. 1). All other variables included 0 within the range of their confidence intervals (Table 2) and were not important contributors to variation in NDI scores.

\section{Discussion}

We quantified variation in nest defense behavior in a population of gray catbirds breeding in Eastern Pennsylvania. Our results indicated that NDI and response time between males and females in this population were not different. Furthermore, not only were NDI and response time the same between paired males and females, they were highly correlated. Nest fate did not appear to be related to levels of NDI or response time. Finally, we found evidence that catbird nest defense varied with characteristics of the offspring that were being defended because our multivariate analysis indicated NDI declined with clutch initiation. Other measures of offspring value (i.e., offspring age and number) were not related to how aggressively catbirds defended their nests. 
Table 1 Results of model selection evaluating variation in nest defense intensity and offspring and individual characteristics in gray catbirds breeding in Eastern Pennsylvania between 2017 and 2018. We used general linear models and included sex, brood (first or later), and stage (incubation or nestling) as fixed factors, and clutch initiation (date of first egg laid, May $1=1$ ), date (observation date, May $1=1$ ), nest day (where day first egg laid $=0$ ), bill length and tail length, and body condition were included as covariates. Except for the null, models shown are only those with a $\Delta \mathrm{AIC}_{\mathrm{c}}<2$

\begin{tabular}{|c|c|c|c|c|c|}
\hline Model & $\mathrm{AIC}_{\mathrm{c}}$ & $\Delta \mathrm{AIC}_{\mathrm{c}}$ & Likelihood & Weight & $\mathrm{K}$ \\
\hline Clutch initiation & 111.63 & 0 & 1 & 0.04 & 3 \\
\hline Clutch initiation + body condition & 113.07 & 1.43 & 0.49 & 0.02 & 4 \\
\hline Clutch initiation + tail length & 113.14 & 1.51 & 0.47 & 0.02 & 4 \\
\hline Clutch initiation + date & 113.16 & 1.53 & 0.47 & 0.02 & 4 \\
\hline Clutch initiation + nest worth & 113.39 & 1.76 & 0.42 & 0.02 & 4 \\
\hline Clutch initiation + stage & 113.45 & 1.82 & 0.4 & 0.02 & 4 \\
\hline Clutch initiation + stage + brood & 113.55 & 1.92 & 0.38 & 0.02 & 5 \\
\hline Clutch initiation + nest day & 113.61 & 1.98 & 0.37 & 0.02 & 4 \\
\hline Intercept only & 116.37 & 4.74 & - & - & 2 \\
\hline
\end{tabular}

\section{Differences in nest defense between the sexes}

Most studies conclude that males defend nests more aggressively than females (Redmond et al. 2009; Klvanova et al. 2011; Svagelj et al. 2012; Shew et al. 2016) and this difference is attributed to the larger size of males compared with females in many species. Larger individuals should be able to defend nests either more efficiently or aggressively with less risk than their smaller counterparts (Wallin 1987). Conversely, if body size and maneuverability are inversely related (Pennycuick 1972; Lima 1986), then nest defense may be less risky for the smaller sex (e.g., Wiklund and Stigh 1983; Andersson and Wiklund 1987). Although male and female catbirds from our study site do not differ in body mass, tarsus length, or bill dimensions, males do have longer wings and tails than females (LJR unpublished data). The larger flight surface relative to body size provided by the longer wings and tails of male catbirds should make them more maneuverable and less prone to injury while defending their nest (e.g., Wiklund and Stigh 1983; Andersson and Wiklund 1987; Kis et al. 2000).
However, based on our measure of nest defense intensity, there was no difference in how aggressively males and females defended their offspring. One explanation for the lack of difference between the sexes is that aggression is a cue used by individuals to evaluate the suitability of a mate thus leading to assortative mating (Andersson 1994). A second explanation is that parents adjust NDI based on the response of their mate (Andersson et al. 1980; Weatherhead 1989). Both explanations predict a correlation between male and female NDI, which is consistent with our findings and thus we are unable to separate between the two hypotheses in the current study.

An alternative explanation to the lack of difference between male and female NDI is the possibility that males did not respond as vigorously as females because of uncertainty in paternity due to extra-pair mating. Reduced paternity has been linked to reductions in male parental care (Dixon et al. 1994; Freeman-Gallant 1996; but see Whittingham et al. 1992), including nest defense by males (Weatherhead et al. 1994; Dolan et al. 2007). In a different population of catbirds, 25\% of broods contain at least one extra-pair young (Smith et al.
Table 2 Model-averaged slope coefficients, standard errors, and 95\% confidence intervals (CI) for offspring and individual variables and their effect on nest defense intensity in gray catbirds in Eastern Pennsylvania. Variables and parameters in italic are those deemed significant because of 95\% confidence estimates that did not include zero

\begin{tabular}{lccc}
\hline Variable & Model-averaged slope coefficient \pm SE & Lower 95\% CI & Upper 95\% CI \\
\hline Clutch initiation & $-0.01 \pm 0.01$ & -0.02 & -0.01 \\
Date & $0.01 \pm 0.05$ & -0.01 & 0.01 \\
Nest day & $0.01 \pm 0.01$ & -0.01 & 0.01 \\
Stage & $-0.01 \pm 0.09$ & -0.18 & 0.17 \\
Brood & $0.07 \pm 0.11$ & -0.15 & 0.28 \\
Nest worth & $0.01 \pm 0.02$ & -0.03 & 0.04 \\
Sex & $-0.01 \pm 0.06$ & -0.13 & 0.12 \\
Bill length & $-0.01 \pm 0.03$ & -0.06 & 0.05 \\
Tail length & $-0.01 \pm 0.01$ & -0.02 & 0.01 \\
Body condition & $-0.01 \pm 0.01$ & -0.04 & 0.02 \\
\hline
\end{tabular}


Fig. 1 Relationship between clutch initiation (May 1=1) and nest defense intensity $\left(\log _{10}\right)$ in gray catbirds breeding on and around the Penn State Schuylkill campus in Eastern Pennsylvania, USA

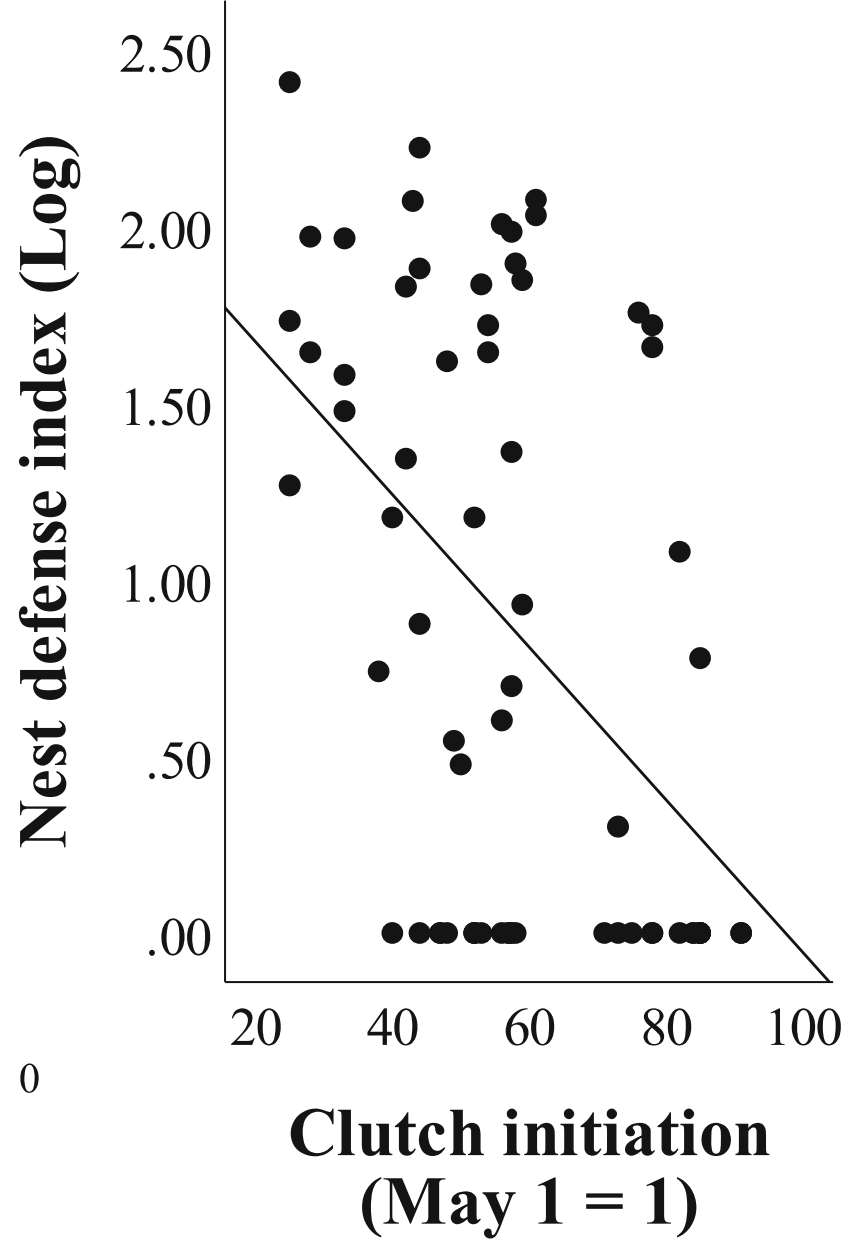

design only captures a small part of the series of events that likely occur during a nest predation event. Adults responding during our trials are immediately presented with a nest predator within one meter of the nest. Although approaching predators could remain undetected by adults until they are within such close proximity of the nest, this seems unlikely. Instead, during a real predation event, adults would have the chance to detect and respond to predators while they are much farther from the nest, allowing them to perhaps use other types of behaviors (e.g., distraction) to prevent the predator from finding the nest or deter it from approaching. Studies, such as ours, that simulate a nest predation event with a model predator do not include the full sequence of events and may fail to detect a relationship between nest defense and nest success.

\section{Offspring value and nest defense}

Because larger broods represent an increase in fitness gains for adults (Newton 1989), NDI is expected to scale with brood size. However, results are mixed in that some species exhibit the expected relationship between nest defense and brood size (Redondo 1989; Wiklund 1990; Olendorf and Robinson 2000) while others do not (Tryjanowski and Golawski 2004; 
Redmond et al. 2009). Offspring age has also been identified as an important contributor to variation in nest defense (Wallin 1987; Redmond et al. 2009; Svagelj et al. 2012). However, nest defense in catbirds was independent of both brood size and offspring age. Instead, our analyses indicated that NDI was inversely related to clutch initiation. Although there is no information related to fledging date and juvenile survival in this species (see Smith et al. 2011), it seems unlikely that variation in juvenile survival is any different in catbirds than other species and catbirds that fledge earlier in the breeding season are more likely to survive into the following year (e.g., Naef-Daenzer et al. 2001; Smith 2004; Shutler et al. 2006). Therefore, from the perspective of an adult catbird, the most important variable related to the value of offspring that drives variation in NDI is the likelihood of young recruiting into the population. The renesting potential (Weatherhead 1979) and positive reinforcement hypotheses (Knight and Temple 1986b) both predict a positive relationship between nest defense and date, thus neither was supported by our data. Catbirds readily replace failed nests and, on our study site, have had as many as four nests in a year and bred well into late-August (Redmond, unpublished). With such a long breeding season, renesting potential likely declines slowly and may not be an important driver of variation in nest defense intensity. The positive reinforcement hypothesis represents more of a methodological concern that we may have avoided by using a model of a predator that represents a threat to both catbird nests and adults.

Acknowledgments We would like to thank the Penn State Schuylkill Campus Advisory Board for allowing use of the land on our study site. Duncan Noble and Donovan Ricco both provided valuable assistance in the field. Brenna E. Traver and two anonymous reviewers gave helpful comments to earlier versions of this manuscript.

Funding information This study was supported in part by funds awarded to LJR through the Penn State Schuylkill Research and Development Grant and the Weist Student Research Endowment from the Penn State Schuylkill Campus Advisory Board.

\section{Compliance with ethical standards}

Conflict of interest The authors declare that they have no conflict of interest.

Ethical approval This study complies with all relevant laws of the United States of America and the state of Pennsylvania. All procedures were approved by the Pennsylvania State University Institutional Animal Care and Use Committee.

Open Access This article is licensed under a Creative Commons Attribution 4.0 International License, which permits use, sharing, adaptation, distribution and reproduction in any medium or format, as long as you give appropriate credit to the original author(s) and the source, provide a link to the Creative Commons licence, and indicate if changes were made. The images or other third party material in this article are included in the article's Creative Commons licence, unless indicated otherwise in a credit line to the material. If material is not included in the article's Creative Commons licence and your intended use is not permitted by statutory regulation or exceeds the permitted use, you will need to obtain permission directly from the copyright holder. To view a copy of this licence, visit http://creativecommons.org/licenses/by/4.0/.

\section{References}

Andersson M (1994) Sexual selection. Princeton University Press, Princeton

Andersson M, Wiklund CG (1987) Sex role partitioning during offspring protection in the rough-legged buzzard Buteo lagopus. Ibis 129: 103-107

Andersson M, Wiklund CG, Rundgren H (1980) Parental defence of offspring: a model and an example. Anim Behav 28:536-542

Arnold TW (2010) Uninformative parameters and model selection using Akaike's information criterion. J Wildlife Manage 74:1175-1178

Boucher DH (1977) On wasting parental investment. Am Midl Nat 111: 786-788

Boukhriss J, Selmi S (2010) Risk-taking by incubating rufous bush robins Cercotrichas galactotes: season-dependent incubation stage effect. J Ethol 28:331-337

Brunton DH (1990) The effects of nesting stage, sex, and type of predator on parental defense by killdeer (Charadrius vociferus): testing models of avian parental defense. Behav Ecol Sociobiol 26:181-190

Burnham KP, Anderson DR (2002) Model selection and multimodel inference: a practical information-theoretic approach, 2nd edn. Springer-Verlag, New York

Clutton-Brock TH (1991) The evolution of parental care. Princeton University Press, Princeton

Curio E, Onnebrink H (1995) Brood defense and brood size in the great tit (Parus major): a test of a model of unshared parental investment. Behav Ecol 6:235-241

Davis HT, Long AM, Campbell TA, Morrison ML (2018) Nest defense behavior of greater roadrunners (Geococcyx californianus) in South Texas. Wilson J Ornithol 130:788-792

Dawkins R, Carlisle TR (1976) Parental investment, mate desertion and a fallacy. Nature 262:131-133

Dixon A, Ross D, O’Malley SLC, Burke T (1994) Paternal investment inversely related to degree of extra-pair paternity in the reed bunting. Nature 371:698-700

Dolan AC, Murphy MT, Redmond LJ, Sexton K, Duffield D (2007) Extra-pair paternity and the opportunity for sexual selection in a socially monogamous passerine. Behav Ecol 18:985-993

Drilling NE, Thompson CF (1988) Natal and breeding dispersal in house wrens (Troglodytes aedon). Auk 105:480-491

Freeman-Gallant CR (1996) DNA fingerprinting reveals female preference for male parental care in Savannah sparrows. Proc R Soc Lond B 263:157-160

Gottfried BM (1979) Anti-predator aggression in birds nesting in old field habitats: an experimental analysis. Condor 81:251-257

Graham KF, Shutler D (2019) How robust are risk-taking associations in incubating birds? A test and a review. Behav Ecol Sociobiol 73:79

Hanssen SA, Hasselquist D, Folstad I, Erikstad KE (2005) Cost of reproduction in a long-lived bird: incubation effort reduces immune function and future reproduction. Proc R Soc Lond B 272:1039-1046

Jonsson P, Agrell J, Koskela E, Mappes T (2002) Effects of litter size on pup defence and weaning success of neighbouring bank vole females. Can J Zool 80:1-5

Kis J, Liker A, Szekely T (2000) Nest defence by lapwings: observations on natural behaviour and an experiment. Ardea 88:155-163 
Klvanova A, Horakova D, Exnerova A (2011) Nest defence intensity in house sparrows Passer domesticus in relation to parental quality and brood value. Acta Ornithol 46:45-52

Knight RL, Temple SA (1986a) Nest defence in the American goldfinch. Anim Behav 34:887-897

Knight RL, Temple SA (1986b) Why does intensity of avian nest defense increase during the nesting cycle? Auk 103:318-327

Krystofkova M, Haas M, Exnerova A (2011) Nest defense in blackbirds Turdus merula: effect of predator distance and parental sex. Acta Ornithol 46:55-63

Lima SL (1986) Predation risk and unpredictable feeding conditions: determinants of body mass in birds. Ecology 67:377-385

Montgomerie RD, Weatherhead PJ (1988) Risks and rewards of nest defence by parent birds. Q Rev Biol 63:167-187

Morrell N, Johnson KM, Tarwater CE, Arcese P (2016) Nest defense and parental investment in song sparrows (Melospiza melodia). Can J Zool 94:473-477

Naef-Daenzer B, Widmer F, Nuber M (2001) Differential post-fledging survival of great and coal tits in relation to their condition and fledging date. J Anim Ecol 70:730-738

Newton I (1989) Lifetime reproduction in birds. Academic Press, London

Olendorf R, Robinson SK (2000) Effectiveness of nest defence in the Acadian flycatcher Empidonax virescens. Ibis 142:365-371

Pavel V, Bures S (2008) Nest defence in the meadow pipit Anthus pratensis: the influence of renesting potential. J Ethol 26:367-373

Pennycuick CJ (1972) Animal flight. Arnold Press, London

Redmond LJ, Murphy MT, Dolan AC, Sexton K (2009) Parental investment theory and nest defense by Eastern Kingbirds. Wilson J Ornithol 121:1-11

Redondo T (1989) Avian nest defence: theoretical models and evidence. Behaviour 111:161-195

Regelmann K, Curio E (1986) Why do great tit (Parus major) males defend their brood more than females do? Anim Behav 34:12061214

Shew JJ, van der Merwe J, Schauber EM, Tallitsch BK, Nielsen CK (2016) A classic question revisited in the red-winged blackbirds: disentangling confounding hypotheses surrounding parental investment theory and nest defense intensity. Behav Ecol Sociobiol 70: 1843-1856

Shutler D, Clark RG, Fehr C, Diamond AW (2006) Time and recruitment costs as currencies in manipulation studies on the costs of reproduction. Ecology 87:2938-2946

Smith HG (2004) Selection for synchronous breeding in the European Starling. Oikos 105:301-311
Smith RJ, Hatch MI, Cimprich DA, Moore FR (2011) Gray catbird (Dumetella carolinensis), version 2.0. In: Poole AF (ed) The birds of North America. Cornell Lab of Ornithology, Ithaca https:// birdsna.org/Species-Account/bna/species/grycat. Accessed July 2019

SPSS (2017) Statistics for windows, V. 25.0. IBM Corp., Armonk

Stickel LF, Stickel WH, Schmid FC (1980) Ecology of a Maryland population of black rat snakes (Elaphe o. obsoleta). Am Midl Nat 103: $1-14$

Svagelj WS, Trivellini MM, Quintana F (2012) Parental investment theory and nest defence by Imperial shags: effects of offspring number, offspring age, laying date and parent sex. Ethology 118:251-259

Thompson FR III, Dijak W, Burhans DE (1999) Video identification of predators at songbird nests in old fields. Auk 116:259-264

Trivers RL (1972) Parental investment and sexual selection. In: Campbell B (ed) Sexual selection and the descent of man. Aldine, Chicago

Tryjanowski P, Golawski A (2004) Sex differences in nest defence by the red-backed shrike Lanius collurio: effects of offspring age, brood size, and stage of breeding season. J Ethol 22:13-16

Verhulst S, van Balen JH, Tinbergen JM (1995) Seasonal decline in reproductive success of the great tit: variation in time or quality? Ecology 76:2392-2403

Visser ME, Lessells CM (2001) The costs of egg production and incubation in great tits. Proc R Soc Lond B 268:1271-1277

Wallin K (1987) Defence as parental care in tawny owls (Strix aluco). Behaviour 102:213-230

Weatherhead PJ (1979) Do savannah sparrows commit the Concorde fallacy? Behav Ecol Sociobiol 5:373-381

Weatherhead PJ (1989) Nest defence by song sparrows: methodological and life history considerations. Behav Ecol Sociobiol 25:129-136

Weatherhead PJ, Montgomerie R, Gibbs HL, Boag PT (1994) The cost of extra-pair fertilizations to female red-winged blackbirds. Proc R Soc Lond B 258:315-320

Whittingham LA, Taylor PD, Robertson RJ (1992) Confidence of paternity and male parental care. Am Nat 139:1115-1125

Wiklund GC (1990) Offspring protection by merlin (Falco columbarius) females; the importance of brood size and expected offspring survival for defense of young. Behav Ecol Sociobiol 26:217-223

Wiklund CG, Stigh J (1983) Nest defence and evolution of reversed sexual size dimorphism in snowy owls Nyctea scandiaca. Ornis Scand 14:58-62

Publisher's note Springer Nature remains neutral with regard to jurisdictional claims in published maps and institutional affiliations. 